\title{
Territorialität IN DER EU-KOHÄSIONSPOLITIK Eine wechselhafte Geschichte: von der Reform der 1980er Jahre zur Post-Lissabon-Ära
}

\author{
Markus GRUBER, Graz*
}

mit 3 Abb. und 1 Tab. im Text

\section{INHALT}

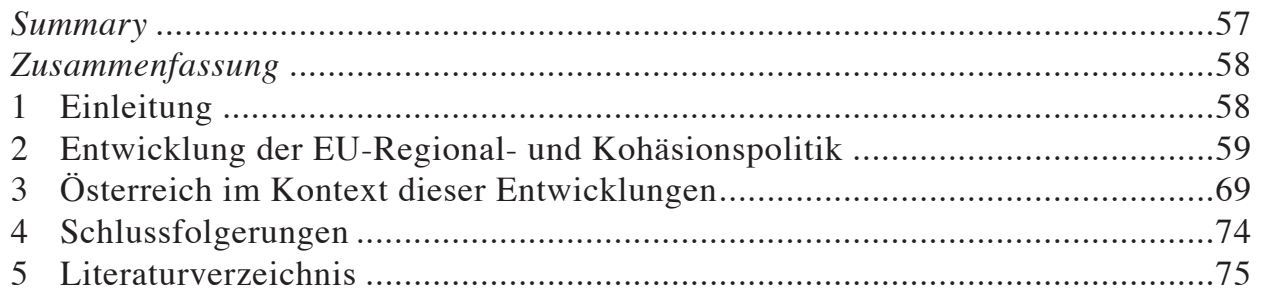

\section{Summary}

Territoriality in EU Cohesion Policy. An oscillating story: From the reforms of the 1980s to the post-Lisbon era

In this article the changing nature of territoriality beyond the programs of European Territorial Cooperation (ETC) will be discussed. It is argued that the various reforms of the EU Cohesion Policy led to a gradual withdrawal of the territorial focus. This resulted in a subordination of the EU Cohesion Policy under the horizontal objectives of competitiveness and growth in the Lisbon era. Various countermovement within the EU member states and the inclusion of the objective of „Territorial Cohesion“ in the EU Treaty entailed a partial return of "Territoriality" in the current period 2014-2020. However, contradictions between cohesion at a large-scale European level, the instrumentalisation of the EU Cohesion Policy for Europe 2020 Objectives and a territorial-, regional- and local-oriented development approach are still persistent. In this context, the article traces the trajectories of the EU Cohesion Policy and their changes and outlines the corresponding reaction in Austria.

\footnotetext{
* Mag. Markus Gruber, convelop - cooperative knowledge design Graz, Bürgergasse 8-10, A-8010 Graz;
} E-Mail: markus.gruber@convelop.at, http://www.convelop.at 


\section{Zusammenfassung}

Dieser Artikel diskutiert die wechselhafte Geschichte der Territorialität in der EUKohäsionspolitik jenseits der Programme der Europäischen Territorialen Zusammenarbeit (ETZ). Es wird dargestellt, dass in den Reformschritten seit 1989 sukzessive eine territoriale Sichtweise zurückgenommen wurde. Dies mündet in der Lissabon-Ära des letzten Jahrzehntes in einer weitgehenden Unterordnung des Aspektes der Territorialität unter die horizontalen Ziele der EU von Wettbewerbsfähigkeit und Wachstum. Erst durch diverse Gegenbewegungen der EU-Mitgliedsstaaten und der Aufnahme des Zieles der „territorialen Kohäsion“ in dem EU-Vertrag erfolgt die Wiederkehr einer stärker territorialen Perspektive in der Periode 2014-2020. Widersprüche zwischen großräumiger Kohäsion auf europäischer Ebene, der Instrumentalisierung der EUKohäsionspolitik für die Europa-2020-Ziele und territorial, regional und lokal orientierten Entwicklungsansätzen erscheinen jedoch keineswegs als aufgelöst. Vor diesem Hintergrund zeichnet dieser Beitrag die Entwicklung der EU-Regionalpolitik im Hinblick auf „Territorialität“ nach und umreißt die entsprechenden Reaktionen Österreichs.

\section{Einleitung}

Die Strukturpolitik der Europäischen Union (EU) beeinflusst maßgeblich die regionalpolitischen Agenden und damit die Entwicklungen der Regionen in Europa. In der Programmperiode 2007-2013 wurde durch die enge Koppelung der Strukturförderung an den Lissabon-Prozess für Wachstum und Beschäftigung und die Vorgabe strategischer Multilevel-Governance-Prozesse ein Paradigmenwechsel in der EU-Regionalpolitik vollzogen. Trotz der Aufwertung der Europäischen Territorialen Kooperation haben in den großen EU-kohäsionspolitischen Programmen der Lissabon-Ära die räumlichen Komponenten zugunsten horizontaler Ziele wie Wettbewerbsfähigkeit und Wachstum an Bedeutung verloren, und es wurden die kleinräumigen Gebietsabgrenzungen aufgegeben. Erst mit der Aufnahme des Ziels der „Territorialen Kohäsion“ in den EUVertrag kommt es wieder zu einer über die Europäische Territoriale Zusammenarbeit hinausgehende Stärkung der territorialen Dimension in den kohäsionspolitischen Programmen der Europäischen Union.

In der neuen EU-Programmperiode 2014-2020 werden Mechanismen vorgesehen, die eine stärkere Beachtung integrierter territorialer Entwicklung sicherstellen sollen. Dies umfasst eine klare Interventionslogik basierend auf (territorialen) Analysen, verstärkte Ergebnisorientierung und eigene Instrumente für die Förderung territorialer Ansätze. Allerdings sind diese Vorgaben in Teilen für die Mitgliedsstaaten fakultativ, d.h. sie sind lediglich ein Angebot an neuen Instrumenten, deren Einsatz in den EU-kohäsionspolitischen Programmen nicht verpflichtend ist. Hier wird erst eine Auswertung der aktuellen Programmgeneration zeigen, in welchem Ausmaß diese tatsächlich von den Mitgliedsstaaten aufgegriffen werden.

Der Beitrag zeichnet die grundlegenden Prämissen der EU-Regionalpolitik und deren Veränderung über die letzten Förderperioden hin zur aktuellen Periode nach und skizziert die entsprechenden Reaktionen Österreichs auf die damit verbundenen Herausforderungen. 


\section{Entwicklung der EU-Regional- und Kohäsionspolitik}

\subsection{Ziele und Finanzierungsinstrumente der EU-Regional- und Kohäsionspolitik}

Die europäische Regional- und Kohäsionspolitik entstand aus drei Stoßrichtungen: erstens, einer Politik die vor allem landwirtschaftlichen Herausforderungen begegnen will, zweitens einer unterstützenden Politik für jene Regionen, die massiv mit industriellem Strukturwandel zu kämpfen hatten und einen Ausgleich zum landwirtschaftlichen Fokus der Regionalpolitik forderten sowie drittens dem Kohäsionsgedanken, der sich mit der Zeit zum dominanten Beweggrund entwickelte. In Titel XVII des EG-Vertrags „Wirtschaftlicher und sozialer Zusammenhalt" ist das gemeinschaftliche Ziel verankert, die Unterschiede bei den regionalen Entwicklungsständen sowie den Rückstand der am stärksten benachteiligten Gebiete, einschließlich der ländlichen Gebiete, zu verringern. Zurückzuführen war dies auf die wachsenden Disparitäten zwischen den europäischen Regionen. Dies wurde als Gefahrenpotenzial für den europäischen Zusammenhalt und die europäische Wettbewerbsfähigkeit wahrgenommen.

Ein kurzer Abriss über die Entwicklung der EU-Kohäsionspolitik zeigt die schrittweise Genese der heutigen Politikkonzeption (zu den Zielen der Finanzierungsfonds vgl. Abb. 1):

- Bereits im Zuge der Bildung der Europäischen Wirtschaftsgemeinschaft (EWG) 1957 in Rom wurden zwei europäische Finanzierungsinstrumente von regionalpolitischer Bedeutung geschaffen: die Europäische Investitionsbank (EIB) sowie der Europäische Sozialfonds (ESF). ${ }^{1)}$ Von einer gemeinsamen Regional- oder Kohäsionspolitik war allerdings nur indirekt die Rede. Lediglich in der Präambel des Vertrags wurde das Bestreben verankert, eine harmonische Entwicklung zu fördern und den Rückstand weniger begünstigter Gebiete zu verringern.

- Mit der Vereinbarung über die Gemeinsame Agrarpolitik (GAP) wurde im Jahr 1962 der Europäische Fonds für landwirtschaftliche Entwicklung (EAGFL) eingerichtet. Dieser wurde kurz darauf in eine Abteilung „Garantie“ und eine Abteilung „Ausrichtung“ unterteilt, wobei die Abteilung „Ausrichtung“ (EAGFL-A) für die Strukturreform der Landwirtschaft sowie für neue Formen ländlicher Entwicklung zuständig und damit von regionalpolitischer Bedeutung war.

- Im Jahr 1975, kurz nach dem Beitritt des Vereinigten Königreichs, Irlands und Dänemarks, wird der Europäische Fonds für regionale Entwicklung (EFRE) begründet. Dieser war anfangs noch vorwiegend auf die Unterstützung der Industriegebiete mit rückläufiger Entwicklung im Vereinigten Königreich ausgerichtet und damit auch auf die Schaffung eines finanziellen Ausgleichs für das Vereinigte Königreich, das nur von geringen Leistungen aus der GAP profitiert(e). Erst schrittweise werden auch Regionen mit generellem Entwicklungsrückstand in das EFRE-Regime einbezogen (nach dem Beitritt Griechenlands, Spaniens und Portugals in den 1980er Jahren).

1) Der ESF hatte von Anfang an seine Bestimmung in der Verminderung struktureller Disparitäten in Europa und fokussiert immer stärker auf die Förderung der Humanressourcen. 
ESF: Erhöhung der Chancen von Menschen auf dem Arbeitsmarkt sowie Schaffung von Arbeitsplätzen und Ausbildungsmöglichkeiten.

EFRE: Stärkung regionaler Wettbewerbsfähigkeit; Förderung des Strukturwandels in industriellen Gebieten und Verringerung regionaler Entwicklungsunterschiede in Europa.

EAGFL-A/ ELER: Förderung der Wettbewerbsfähigkeit ländlicher Gebiete, der Erhaltung und Schaffung von Arbeitsplätzen sowie der Diversifizierung in diesen Gebieten.

Das Finanzinstrument für die Ausrichtung der Fischerei (EMFF) gilt seit 1999 als Strukturfonds. Es kommt in Österreich nur sehr eingeschränkt zur Anwendung.

Quelle: Eigene Zusammenstellung

Abb. 1: Überblick über Fonds und ihre Ziele

Erst etliche Jahre später (1987) werden die regionalpolitischen Zielsetzungen auch formal festgelegt: Im Zuge der Einheitlichen Europäischen Akte wird ein neuer Titel, „der wirtschaftliche und soziale Zusammenhalt" in den EWG-Vertrag integriert (Art. 130a-130e). Damit wurden die einst unverbindliche Zielsetzung der EWG-Präambel auch rechtlich verankert und der Grundstein für die solidarischen regionalpolitischen Agenden der Union gelegt.

\subsection{Reformschritte der europäischen Kohäsions- und Regionalpolitik (vgl. Abb. 2)}

\section{Grundlegende Reform der Strukturfonds 1989}

Die EU-Kohäsionspolitik war bis zur Reform 1989 ein überwiegend unspezifischer, auf Einzelprojektförderungen aufbauender Politikansatz. Mit der Strukturfondsreform 1989 wurde die EU-Regionalpolitik auf eine neue Grundlage gestellt und programmatisch ausgerichtet. Die drei bislang etablierten Fonds ESF, EAGFL und EFRE wurden aufeinander abgestimmt novelliert und als eine, zusammengehörige Aktion der

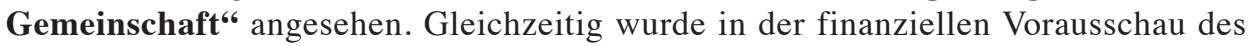
„Delors-Pakets“ eine Verdoppelung der Strukturausgaben zwischen 1988 und 1993 auf $31 \%$ der Gemeinschaftsausgaben beschlossen. Die Schwerpunkte der Reform sahen Folgendes vor:

- Prinzip der mehrjährigen Programmplanung - mehrjährige (Regional)-Programme als Voraussetzung für die Vergabe von Strukturfondsmitteln;

- Konzentration der Mittel auf vorrangige Ziele mit Bevölkerungsobergrenzen und unterschiedlichen Förderintensitäten;

- Dezentralisierung der Einzelantragsentscheidungen;

- Partnerschaft bei Entwicklung und Umsetzung der regionalen Entwicklungsprogramme zwischen der Europäischen Gemeinschaft, Mitgliedsstaaten und den Wirtschafts- und Sozialpartnern;

- Prinzip der Additionalität, wonach der Einsatz der EU-Strukturfondsmittel zusätzlich zu den nationalen Mitteln erfolgt und diese nicht ersetzen dürfen. 


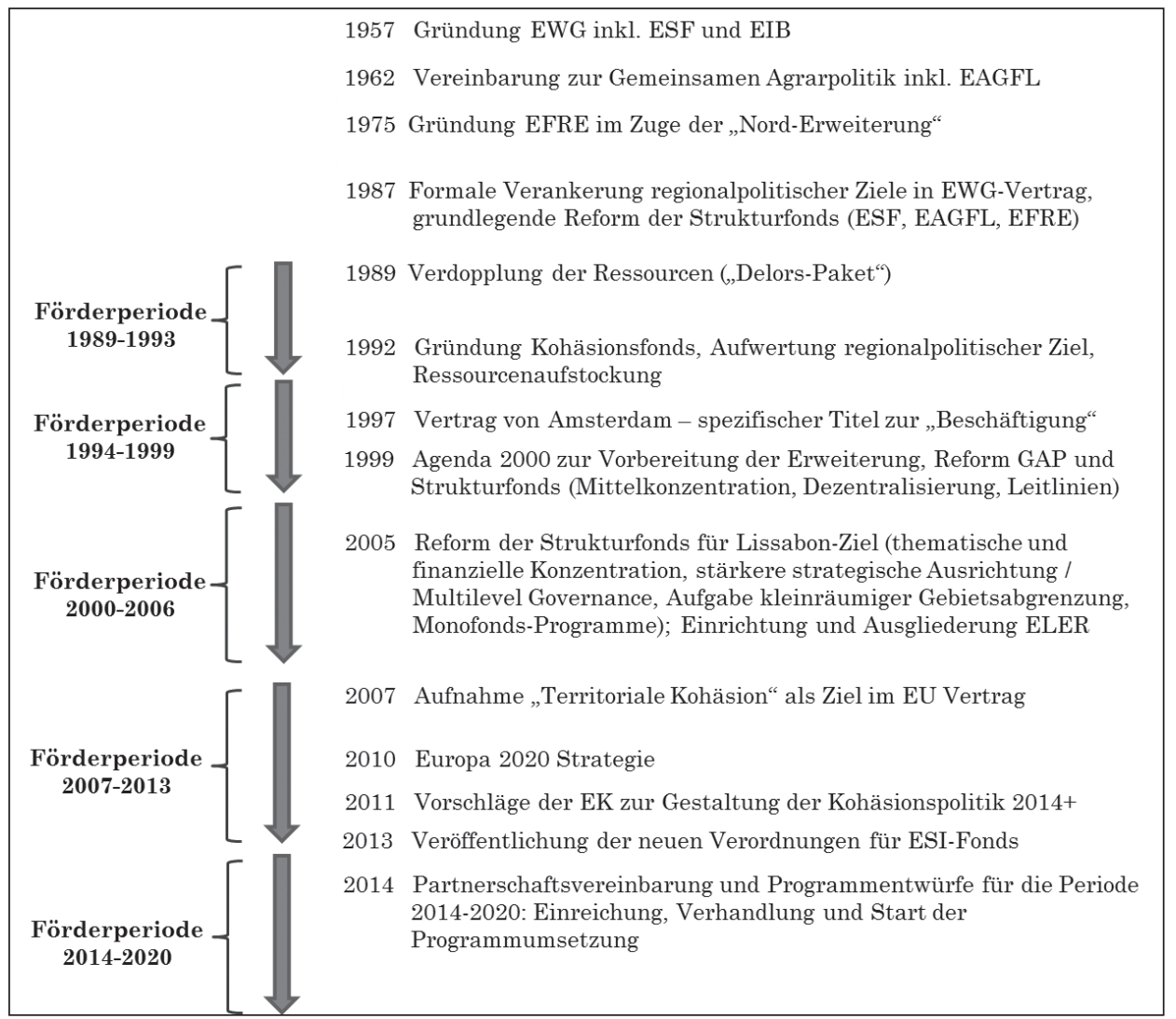

Quelle: Aktualisiert nach Gruber \& PoHn-WeIdinger 2012

Abb. 2: Entwicklung der europäischen Strukturfonds

Insbesondere das Prinzip der Programmplanung zeigt bis heute deutliche Auswirkungen für die Regionen. Für jedes Ziel bzw. Zielgebiet muss ein Programm erstellt werden, das eine Bewertung der aktuellen Lage, die Programmziele, -strategie, -schwerpunkte, -finanzierung, Angaben zur Programmabwicklung sowie eine Ex-anteBewertung des Programms enthält.

\section{Territoriale Dimension in der EU-Kohäsionspolitik}

Mit den regionalen Entwicklungsprogrammen wurden ein programmatischer Ansatz und ,Territorialität" in die EU-Kohäsionspolitik eingeführt. Versteht man unter „Territorialität“, dass raum- oder regionsspezifische Entwicklungsherausforderungen durch aufeinander abgestimmte Maßnahmen angegangen und Potenziale aktiviert werden, so war die EU-Kohäsionspolitik stark räumlich-territorial orientiert.

Die Zielsetzungen im Bereich des Europäischen Fonds für regionale Entwicklung (EFRE) waren auf räumliche Problemgebiete ausgerichtet wie zum Beispiel „Förde- 
rung von Regionen mit Entwicklungsrückstand“, ,Förderung von Industriegebieten mit rückläufiger Entwicklung“ oder „Förderung von ländlichen Gebieten“. Der Anspruch folgte einer integrierten, sektor- und fondsübergreifenden Entwicklung, indem die verschiedenen Fonds als Finanzierungsinstrumente in einem Programm kombiniert werden konnten. Daneben verfolgte der ESF weitgehend nationale Zielsetzungen wie die Anpassung der Arbeitskräfte an den industriellen Wandel.

Ergänzend wurden sogenannte Gemeinschaftsinitiativen ${ }^{2)}$ eingesetzt, die neben horizontalen Zielen wie zum Beispiel der Entwicklung von kleinen und mittleren Unternehmen (KMU-Initiative) vorwiegend auf räumliche Problemgebiete in den Mitgliedsstaaten ausgerichtet waren. Anzuführen sind hier beispielsweise die Umstellung von Textilregionen - RETEX, von Stahlregionen - RESIDER, von Kohlenregionen - RECHAR, die bottom-up-orientierte Entwicklung im ländlichen Raum - LEADER und später im städtischen Bereich - URBAN. Als eines der wichtigsten Instrumente zur Förderung der territorialen Kohäsion auf europäischer Ebene ist die im Jahr 1990 eingeführte Gemeinschaftsinitiative INTERREG anzusehen, mit deren Hilfe sich die EU die Aufgabe gestellt hat, die Regionen auf eine Gemeinschaft ohne interne Grenzen vorzubereiten und strukturelle Probleme von Grenzgebieten zu behandeln.

In der Folge kam es regelmäßig zu Reformschritten, hervorgerufen durch wiederkehrende Diskussionen über die Effektivität der EU-Kohäsionspolitik. Sie waren vorwiegend geprägt durch Fragen der Konzentration des Mitteleinsatzes und einer höheren Kohärenz der EU-Kohäsionspolitik mit weiteren EU-Politikfeldern und führten letztlich zu einer schrittweisen De-Territorialisierung der EU-Kohäsionspolitik.

\section{Agenda 2000 - Vorbereitung auf die Erweiterung}

Ein nächster Meilenstein war die politische Einigung über die Agenda 2000. Diese umfasst ein großes Reformprogramm zur Stärkung der Gemeinschaftspolitiken, um den neuen Herausforderungen durch die Erweiterung der Union um zehn neue Mitgliedsstaaten im Jahr 2004 Rechnung zu tragen. Dabei wurde unter anderem die Gemeinsame Agrarpolitik (stärkere Berücksichtigung von Umweltzielen und Betonen der Aufgabe der Landschaftspflege etc.) sowie die europäische Strukturpolitik (bessere Effizienz durch Mittelkonzentration, Zuständigkeitsklärungen/Dezentralisierung, Vereinfachen der rechtlichen Grundlagen mit einer allgemeinen Verordnung für die gemeinsamen Grundsätze und vorrangigen Ziele sowie spezifischen Verordnungen für die einzelnen Fonds etc.) reformiert - beides von regionalpolitischer Relevanz.

Konkret führte dies zu einer deutlichen Reduktion der an räumlichen Gebietstypen und -problemlagen orientierten Zielsetzungen und einer stärker strategischen Orientierung der kohäsionspolitischen Programme. Diese wurden zusammengefasst und auf Ziele ausgerichtet. Die Zahl der Gemeinschaftsinitiativen wurde reduziert. Übrig geblieben sind INTERREG sowie für die ländlichen Regionen LEADER und im städtischen Bereich URBAN. Die Fragen zur Entwicklung des ländlichen Raumes wurden zunehmend von der Gemeinsamen Agrarpolitik beansprucht, was mit einer Verstärkung des Fokus auf die Agrarwirtschaft einhergeht.

\footnotetext{
2) Gemeinschaftsinitiativen waren Förderungsprogramme in bestimmten Problembereichen, die von der Europäischen Kommission (EK) initiiert und beschlossen wurden.
} 


\section{Orientierung an der Lissabon-Strategie für „Wachstum und Beschäftigung“}

Globalisierung und Wissensökonomie setzten die Europäische Union im letzten Jahrzehnt wirtschaftspolitisch unter Handlungsdruck. So steckte sich der Europäische Rat von Lissabon [Lisboa] im Jahr 2000 bekanntlich das ambitionierte Ziel, der wettbewerbsfähigste und dynamischste Wirtschaftsraum in der Welt zu werden (,Lissabon-Strategie“).

Nach wenig zufriedenstellenden Entwicklungen in den ersten Jahren wurde die Lissabon-Strategie im Jahr 2005 neu ausgerichtet ohne dabei ihre zentralen Zielsetzungen zu verändern: Wettbewerbsfähigkeit sowie Wachstum und Beschäftigung bildeten den Kern der europäischen Politik. Im Zuge der Neuausrichtung wurde die europäische Strukturpolitik als zentrales Umsetzungsinstrument der Lissabon-Strategie hervorgehoben.

Die Reformen waren für die Strukturfonds und ihre Förderansätze von weitreichender Bedeutung. Sie führten zu einem Paradigmenwechsel (vgl. Abb. 3) mit Hauptaugenmerk auf die Steigerung der regionalen Wettbewerbsfähigkeit. Forschung, Entwicklung und Innovationsförderung wurden zur zentralen Säule der Strukturfonds erklärt. Im Einzelnen umfasste die Reform folgende Punkte:

- thematische und finanzielle Konzentration auf wenige Ziele;

- Einführung einer strategischen Multilevel-Governance-Architektur: politische Strategie, Kohäsionsleitlinien, nationale strategische Rahmenpläne, operationelle Programme;

- die Auflösung kleinräumiger Zielgebietsabgrenzungen;

- die Auflösung der Gemeinschaftsinitiativen und deren „Mainstreaming“ in die großen Zielprogramme.

\begin{tabular}{ll}
\hline 2000-2006 „Nationale Kohäsion“ & 2007-2013 „Europäische Kohäsion“ \\
\hline $\begin{array}{l}\text { Stärkung der Wettbewerbsfähigkeit, } \\
\begin{array}{l}\text { Wachstum und Beschäftigung in } \\
\text { strukturschwachen Regionen }\end{array}\end{array}$ & $\begin{array}{l}\text { Stärkung der Wettbewerbsfähigkeit, } \\
\text { Wachstum und Beschäftigung }\end{array}$ \\
\hline Ausgleichsorientierung & Potenzialorientierung \\
\hline Agenda 2000: kleinräumige Gebietsabgrenzung & $\begin{array}{l}\text { "Lissabon-Strategie“: } \\
\text { Wachstum und Beschäftigung }\end{array}$ \\
\hline Leitlinien als Koordination & $\begin{array}{l}\text { Horizontale und vertikale Koordination } \\
\text { (u.a. strategisches Kohäsionskonzept) }\end{array}$ \\
\hline Zielprogramme, Gemeinschaftsinitiativen & $\begin{array}{l}\text { Reduktion auf drei große Ziele } \\
\text { (Monofondsprogramme) }\end{array}$ \\
\hline
\end{tabular}

Quelle: Gruber \& Zumbusch 2008

Abb. 3: Paradigmenwechsel der Strukturfondsperiode 2007-2013

Durch die enge Koppelung der Strukturförderung an den Lissabon-Prozess, durch die Auflösung der kleinräumigen Gebietsabgrenzungen und durch die Vorgabe stra- 
tegischer Multilevel-Governance-Prozesse wurde ein Paradigmenwechsel in der EURegionalpolitik vollzogen: In den nationalen Strukturfondsprogrammen dominiert nun eine großräumigere Potenzial- über die bislang im Vordergrund stehende kleinräumige Ausgleichsorientierung. Das Kohäsions- und Ausgleichsziel wird vor allem im europäischen Maßstab durch Konzentration von mehr als $80 \%$ der Mittel auf die Konvergenzländer in den Erweiterungsländern und südlichen Mitgliedsstaaten weiter forciert. Parallel wurde zudem die Förderung des ländlichen Raums reformiert, der Europäische Landwirtschaftsfonds für die Entwicklung des ländlichen Raums (ELER) eingerichtet und dieser aus den Strukturfonds ausgegliedert.

\section{Von der Lissabon-Strategie zu Europa 2020}

Die im Juni 2010 vom Europäischen Rat beschlossene neue Wachstums- und Beschäftigungsstrategie „Europa 2020“ (EK 2010) ist in wesentlichen Punkten eine Fortsetzung der wachstumsorientierten Vorgängerstrategie. Das deutlich verstärkte „Governance-Konzept“ zielt vor allem auf eine vertiefte Koordination ab - horizontal zwischen den Fonds, vor allem aber auf eine vertikale Koordination von Europa 2020 über den Nationalen Reformplan ${ }^{3)}$ hin zu den EU-kofinanzierten Programmen.

Zur Stärkung der thematischen Integration wurden der 2007 aus den Strukturfonds ausgekoppelte ELER und der Fischereifonds zusammen mit dem EFRE und den ESF wieder unter den Europäischen Struktur- und Investitionsfonds (ESI-Fonds) zusammengefasst. Es wurden elf thematische Ziele definiert, an denen sich alle Fonds in der Umsetzung orientierten müssen. Damit wird der mit der Reform 2005 eingeschlagene Weg der Bindung der Kohäsionspolitik an EU- und nationale Ziele nochmals festgeschrieben. Die EU-Kohäsionspolitik wird noch stärker an die Ziele der übergeordneten Dachstrategie sowie die daraus im Rahmen der Nationalen Reformpläne definierten Ziele gebunden, die keine regionalpolitischen Ziele beinhalten. ${ }^{4)}$ Diese beziehen sich auf folgende Kernziele:

- Erhöhung der Beschäftigungsquote in \%

- Erhöhung der Ausgaben für Forschung und Entwicklung (F\&E) in \% des BIP

- Verringerung des $\mathrm{CO}_{2}$-Ausstoßes

- Steigerung des Anteils erneuerbarer Energie

- Verbesserung der Energieeffizienz durch Verringerung des Energieverbrauchs in Mio. t Rohöleinheiten

- Verringerung der Schulabbrecherquote

- Erhöhung der Zahl der Hochschulabsolventen in \%

- Senkung des Anteils der von Armut und sozialer Ausgrenzung Betroffenen

\footnotetext{
3) Nationale Reformprogramme: Die EU-Mitgliedsstaaten müssen im Rahmen der Umsetzung der Europa2020-Strategie jedes Jahr ein nationales Reformprogramm vorlegen. Darin skizzieren sie ihre Strategie für nachhaltiges Wachstum und solide öffentliche Haushalte.

4) Zudem werden sogenannte Konditionalitäten eingeführt. Das sind „bindende Auflagen“, die von den Mitgliedsstaaten zu erfüllen sind (z.B. das Vorliegen einer Smart-Specialisation-Strategie). Die Konditionalitäten sollen die Effektivität des Einsatzes der Kohäsionsmittel steigern.
} 


\section{Enträumlichung der Lissabon-Ära}

Mittelaufstockungen, formale Reformen und der stete Versuch, größere Konzentration zu erreichen, begleiten die Entwicklung der Strukturfonds über die letzten Jahrzehnte. Dies war neben der Aufwertung des Ziels der Europäischen Territorialen Kooperation mit dem Trend hin zur engen Bindung an vor allem horizontale Politikziele auf europäischer Ebene verbunden. Die Konsequenz lag in einem zunehmenden Rückzug von territorial orientierten Ansätzen in den großen EU-kohäsionspolitischen Programmlinien der EU. Der vorwiegend ausgleichsorientierte Handlungsansatz ist definitiv innovations- und potenzialorientierten Vorgehensweisen gewichen. Das Ausgleichsziel der Regionalpolitik wurde auf die großräumige, europäische Ebene verlagert.

Damit erfolgen architektonische Verschiebungen im Hinblick auf eine EU-Kohäsionspolitik, die auf territoriale, gebietstypische Problemstellungen ausgerichtet sind:

- Steigender Mitteleinsatz bei erweitertem Unionsgebiet und Konzentration der eingesetzten Mittel auf weniger Ziele/Programme. Für 2014-2020 umfasst die EUKohäsionspolitik rund ein Drittel des EU-Budgets (351,8 Mrd. Euro).

- Die inhaltliche Bindung und damit auch Ausrichtung auf übergeordnete politische Dachstrategien mit dem Fokus auf Wettbewerbsorientierung und Wachstum. Die räumlich orientierten Ziele wurden schrittweise zugunsten allgemeiner wirtschaftspolitischer Zielsetzungen aufgegeben.

- Eine wachsende Bedeutung großräumigerer Entwicklungsperspektiven, einhergehend mit einem zunehmenden Fokus auf F\&E und Innovation. Das führte auch dazu, dass neue Akteure wie Hochschulen, Forschung seinrichtungen etc. für die kohäsionspolitischen Programme an Relevanz gewonnen haben.

- Die Auflösung der kleinregionalen Fördergebiete zugunsten einer flächendeckenden Unterstützungsmöglichkeit bedeutet eine stärkere Einbindung von prosperierenden Zentralräumen und städtischen Regionen und führte insgesamt zu einem Verständnis von größeren, zusammenhängenden Regionen.

- Damit verbunden war ein paradigmatischer Wechsel von der Ausgleichs- hin zur Potenzial- und Wettbewerbsorientierung. Die Ausgleichsorientierung wird nur noch auf gesamteuropäischer Ebene verfolgt, indem die Mittel auf die weniger entwickelten Mitgliedsstaaten konzentriert werden.

- Die an Problemlagen und Gebietstypen orientierten Programme wurden damit weitgehend zugunsten nationaler Programme und horizontaler Ziele wie Wettbewerbsfähigkeit und Wachstum zurückgenommen oder überhaupt aus der Kohäsionspolitik ausgekoppelt (z.B. ländliche Räume).

Dies bedeutet, dass sich die Sichtweise auf Regionen und die territoriale Dimension und somit auch die Möglichkeiten für die Gestaltung regionaler Entwicklungsprozesse deutlich verändert haben. An spezifischen Problemlagen ausgerichtete räumliche Programme haben an Bedeutung verloren und „Territorialität“ wird wenn, dann in deutlich größeren räumlichen Zusammenhängen gedacht. Dies veränderte letztlich aber auch völlig die Sichtweise auf die Regionalentwicklung, ihre Regionen, deren Größen und deren Akteure. Dieser schleichenden „De-Territorialisierung“ der Kohäsionspolitik in der Lissabon-Ära steht jedoch als Gegenpol eine Debatte über die „Territoriale Dimension“ gegenüber. 


\subsection{Europäische Debatte zur „Territorialen Dimension“(5)}

\subsubsection{Zwischenstaatliche Kooperation: Die „Territoriale Agenda“}

Zwischen den EU-Mitgliedsstaaten wird das Thema der „Territorialen Dimension“ seit den 1990er Jahren auf politischer Ebene in zumeist losem Rahmen, aber kontinuierlich diskutiert. Die informelle Zusammenarbeit der EU-Mitgliedsstaaten zu Themen der (EU)-Raumentwicklung oder des ,Territorialen Zusammenhalts“ folgt der Tradition des Europäischen Raumentwicklungskonzeptes 1999 (EUREK). Aufgrund der Frage, welchen Beitrag Raumentwicklungspolitik zum Erreichen der Ziele des Lissabon-Prozesses leisten kann, erhielt die Diskussion zur „Territorialen Dimension“ in den ersten Jahren der 2000er Jahre einen neuen Schwung.

Dieser Diskussionsprozess mündete in die sogenannte „Territoriale Agenda“6 (TA 2007). In Kernbereichen wies die TA Ähnlichkeiten zum EUREK auf. Hierzu zählt das Bestreben, die Potenziale der Regionen und Städte für ein nachhaltiges Wirtschaftswachstum und mehr Beschäftigung zu mobilisieren und die Vielfältigkeit der Standorte als Chance zu begreifen. Faktisch blieb die Agenda von geringem politischem Gewicht, da sie einen unverbindlichen Charakter hatte und keine direkten rechtlichen oder finanziellen Konsequenzen nach sich zog.

Ein zur EU-Raumentwicklung komplementärer Prozess der zwischenstaatlichen Zusammenarbeit hat sich seit 1998 zu städtischen Themen etabliert. So wurde 2007 auch die „Leipzig-Charta zur nachhaltigen europäischen Stadt“" angenommen, womit die besondere Rolle der Städte für die weitere europäische Entwicklung und die damit verbundenen Herausforderungen hervorgehoben wurde.

Im Jahr 2011 wurde die Territoriale Agenda 2007 durch die ,Territoriale Agenda der Europäischen Union 2020“ (TA 2020) abgelöst. Basierend auf Experteneinschätzungen (EU 2011a) und im Einklang mit „Europa 2020“ verfolgt die Agenda das Ziel, „die territoriale Dimension auf allen Regierungsebenen stärker in verschiedene Politikbereiche zu integrieren und die Umsetzung der Europa-2020-Strategie im Einklang mit den Grundsätzen des territorialen Zusammenhalts zu gewährleisten“ (EU 2011b). Da auch die Territoriale Agenda 2020 einen unverbindlichen Charakter aufweist, liefert sie in erster Linie EU-weite Grundsätze für die Raum- und Regionalentwicklungspolitik mit langfristiger strategischer Bedeutung.

\subsubsection{Europäische Kommission: Territoriale Kohäsion}

Vor allem durch die Impulse aus dem zwischenstaatlichen Diskussionsprozess wurde die territoriale Dimension in den 2000er Jahren von der Europäischen Kommission (EK) wieder explizit aufgegriffen. Im Lichte der Territorialen Agenda wurde mit dem EU-Vertrag von Lissabon das Ziel des ,Territorialen Zusammenhalts“6 (territorial cohesion) als gemeinsame Zuständigkeit der EU und der Mitgliedsstaaten neu eingeführt. Damit wurde eine aktivere Rolle der EK in der Diskussion notwendig. Unmittelbarer Ausdruck dieser Entwicklung sind das 2008 veröffentlichte Grünbuch

5) Siehe dazu Gruber \& Pohn-Weidinger 2012. 
zum Territorialen Zusammenhalt (EK 2008) sowie die Verankerung des Begriffs in der Strategie „Europa 2020“ im Jahr 2010 (EK 2010). Ziel des Grünbuches war es, die Sichtweise der EK zum Konzept „territorialer Zusammenhalt“ zu erläutern, ihr Verständnis zum Verhältnis zwischen wirtschaftlicher und sozialer Dimension der Kohäsionspolitik darzulegen und eine öffentliche Debatte durch eine Reihe von im Grünbuch aufgeworfenen Fragen anzuregen.

Darin wird als Ziel des territorialen Zusammenhalts definiert, , die harmonische Entwicklung aller Gebiete sicherzustellen und dafür zu sorgen, dass die Bürger die jeweiligen Gegebenheiten dieser Gebiete optimal nutzen können " (S. 3). Territorialer Zusammenhalt wird damit als ressourcenorientiertes Konzept verstanden, in dem die Vielfalt der Regionen als Vorteil und Chance begriffen wird und die Nutzung bestehender Ressourcen zu einer nachhaltigen Entwicklung der gesamten EU beitragen kann. Darüber hinaus wurde auch die Berücksichtigung von Regionen mit speziellen Bedürfnissen stark diskutiert. ${ }^{6)}$

Parallel dazu sind zwei Diskussionsstränge von Interesse:

Eine territoriale Orientierung wurde durch den im Rahmen der Vorbereitung auf die Periode 2014+ von Fabrizio BARCA erstellten unabhängigen Expertenbericht zur Neugestaltung der Kohäsionspolitik (BARCA 2009) gestärkt. BARCA kritisierte die Ausrichtung der Kohäsionspolitik auf die sektoralen wirtschaftlichen Prioritäten. Er weist auf die Wichtigkeit der Abstimmung der Maßnahmen der Wirtschafts- und Territorialpolitik auf die Charakteristika und Bedürfnisse der Regionen hin und empfiehlt eine sogenannte ,place-based policy“. „A place-based policy is a long-term strategy aimed at tackling persistent underutilisation of potential and reducing persistent social exclusion in specific places through external interventions and multilevel governance. It promotes the supply of integrated goods and services tailored to contexts, and it triggers institutional changes." (BARCA 2009, S. VII).

In einem parallelen, von der Organisation für wirtschaftliche Zusammenarbeit und Entwicklung (Organisation for Economic Co-operation and Development, OECD) ausgehenden Diskussionsprozess wurden regionale Innovationsstrategien unter dem Titel „Smart Specialisation“ in einen neuen Kontext gesetzt: Der Smart-SpecialistionAnsatz baut auf den Erfahrungen mit regionalen Innovationsstrategien auf (z.B. Соок et al. 1997, 2000) und entwickelt diese im Hinblick auf die Einbindung von Partnern aus Wissenschaft, Forschung, Wirtschaft, Politik (,entrepreneurial discovery“) sowie eine erhöhte Verbindlichkeit durch Ziele, Monitoring und Zuordnung von Finanzmitteln weiter: „.... Innovation strategies for smart specialisation are integrated, placebased economic transformation agendas ..." (EC 2012). Es wird damit verstärkt auf die Bedeutung des Territoriums für erfolgreiche Innovationsstrategien hingewiesen.

\footnotetext{
6) Konzentration: Nutzen der Vorteile aus wirtschaftlicher und gesellschaftlicher Konzentration unter Vermeidung negativer Agglomerationseffekte; Anbindung der Gebiete untereinander (Raumüberwindung, Zentralität und Peripheralität, Netzinfrastrukturen); den Zugang zu Dienstleistungen von allgemeinem wirtschaftlichem Interesse, die Zusammenarbeit zwischen den Gebieten fördern, um Umwelt- und Strukturprobleme auf der jeweils geeigneten territorialen Ebene zu lösen und die Zusammenarbeit in den grenzüberschreitenden Regionen der neuen Mitgliedsstaaten sowie an den Außengrenzen der EU zu stärken.
} 
Diese sollen sich an regionalen Stärken orientieren. Das Vorliegen von Smart-Specialisation-Strategien wird über die sogenannten Ex-ante-Konditionalitäten bindend für die Inanspruchnahme von EU-Mitteln zur Innovationsförderung.

\subsection{4+: Rückkehr der Territorialen Dimension in die EU-Kohäsionspolitik?}

Die Auseinandersetzung im Rahmen der Vorbereitung zur Neugestaltung der EUKohäsionspolitik 2014+ mit den dortigen Diskussionen zur ,place-based policy“ im Rahmen des BARCA-Berichtes sowie die Aufnahme des dritten EU-Kohäsionsziels der „Territorialen Kohäsion“ hat letztlich einen deutlichen Niederschlag in den Regelungen der Kohäsionspolitik 2014+ gefunden.

Explizit angesprochen wird die Zielsetzung, mit den Fonds den wichtigsten territorialen Herausforderungen zu begegnen. Dabei wird verstärktes Augenmerk auf die Ergebnisorientierung gelegt. Es wird erwartet, dass ausgehend von einer (territorialen) Analyse eine klare Interventionslogik verfolgt wird (vgl. dazu HummelbrunNer et al. 2013). Darin sind die Veränderungsziele zu definieren sowie damit verbundene Ergebnisindikatoren. Die Interventionen müssen aus der (territorialen) Analyse abgeleitet sein. Damit verbunden kann auch die Fokussierung der Mittel auf spezifische Gebiete erfolgen.

Im Hinblick auf die territorialen Gesichtspunkte wird die Rolle von Städten, funktionalen Gebietseinheiten und Regionen mit spezifischen Problemen (u.a. Berggebiete) gesondert erwähnt. In den programmatischen Vereinbarungen zwischen der Europäischen Kommission und den Mitgliedsstaaten ist auf die integrierte territoriale Entwicklung Bezug zu nehmen.

In einem eigenen Abschnitt zu ,,integrierter territorialer Entwicklung“ ist auf die wichtigsten territorialen Entwicklungsbedürfnisse, Engpässe und Potenziale einzugehen, die mithilfe der EU-Mittel angesprochen werden sollen.

Schließlich werden neue Instrumente vorgeschlagen, die integrierte Politiken in speziellen Territorien ermöglichen:

- Integrierte Territoriale Investitionen (ITI) sollen die Steuerung von miteinander in Beziehung stehenden Aktionen aus verschiedenen EFRE- und ESF-Programmen und/oder Prioritäten unterstützen.

- Community Local Lead Development (CLLD) zielen auf eine von lokalen Aktionsgruppen getragene Entwicklung, die eine Ausweitung des LEADER-Ansatzes auf alle Fonds ermöglichen soll.

- Es gibt eine explizite Regelung für integrierte nachhaltige Stadtentwicklung im Rahmen der territorialen Entwicklung. Die Betonung liegt dabei auf einer Stärkung des integrierten Ansatzes, einer besseren Einbindung der Städte und einer Wiedereinführung einer experimentellen Komponente. So sind beispielsweise im EFRE zumindest 5\% der Mittel zweckgebunden für eine integrierte nachhaltige Stadtentwicklung zu verwenden. 
Im Rahmen der neu eingeführten sogenannten ex-ante-Konditionalitäten ist das Vorliegen einer nationalen oder regionalen Smart-Specialisation-Strategie eine Grundvoraussetzung für die Förderung von Forschung und Innovation mithilfe des EFRE-Programmes.

Damit werden nach der De-Territorialisierungstendenz der Lissabon-Ära in der Programmperiode 2014-2020 wieder verstärkt Mechanismen vorgegeben, um eine integrierte territoriale Sichtweise in die kohäsionspolitischen Programme aufzunehmen. Diese sind aber in vielen Bereichen fakultativ. Es bleibt auch die Gefahr der Beschränkung auf verbale Argumentationen in den Programmen, ohne dass diese zu echten substanziellen Änderungen in der Praxis führen. Nach ersten Sichtungen von Programmentwürfen ist jedoch davon auszugehen, dass die territoriale Dimension in erster Linie über die vorgeschriebene städtische Entwicklung abgedeckt wird, während gerade neue Instrumente wie CLLD nur sehr zögerlich aufgegriffen werden. Nichtsdestotrotz kehren damit territorial-räumliche Aspekte - wenn auch nur in Ansatzpunkten - wieder in die Mainstream-Programme der EU-Kohäsionspolitik zurück. Es wird sich erst in einer Zusammenschau der Struktur und Inhalte der neuen Programmgeneration zeigen, ob und in welchem Ausmaß diese über die unmittelbaren Vorgaben für die städtische Entwicklung hinausgehend aufgegriffen werden.

\section{3 Österreich im Kontext dieser Entwicklungen}

Österreich partizipiert an der EU-Kohäsionspolitik seit dem EU-Beitritt im Jahr 1995. Es gilt unter den mit der Regionalförderung befassten Experten als weitgehend geteilte Meinung, dass die EU-Regionalförderung zu einem deutlich stärkeren Fokus der österreichischen Politik auf „Regionen“ führte. Durch den EFRE und Teile des EAGFL-A (später ELER) kam es zu einer Umverteilung von Mitteln zugunsten von regionalen/lokalen Entwicklungsvorhaben. Durch die umfangreichen Gebietsabgrenzungen in der ersten Periode und durch die neu zur Verfügung stehenden Mittel wurde der räumliche Fokus der österreichischen Regionalpolitik deutlich erweitert. Regionalpolitische Initiativen konnten fast flächendeckend auf die österreichischen ländlichen und industriell geprägten Regionen (außerhalb der Zentralräume) ausgeweitet werden (GRUBER 2009).

Aufgrund der föderalen Struktur waren und sind die primäre Umsetzungsebene der regionalpolitischen Programme des EFRE die Länder (umgangssprachlich „Bundesländer“). Die Länder verfügen heute - nicht zuletzt angestoßen durch die EU-Planungserfordernisse - über übergeordnete Landesentwicklungsleitbilder, Wirtschafts- und Innovationsstrategien und institutionell über entsprechende EntwicklungsAgenturen. Die Mittel der Kohäsionspolitik dienen zur Verstärkung und Umsetzung dieser Strategien. Die Ebene der Länder (NUTS-II ${ }^{7)}$ ) erscheint aufgrund der relativ

\footnotetext{
7) NUTS (französisch Nomenclature des unités territoriales statistiques) bezeichnet eine hierarchische Systematik zur eindeutigen Identifizierung und Klassifizierung der räumlichen Bezugseinheiten der amtlichen Statistik in den Mitgliedsstaaten der Europäischen Union.
} 
geringen Größe der österreichischen Länder für eine territoriale Abstimmung gut geeignet. Im Zeitverlauf zeigt sich folgendes Bild (vgl. dazu Tab. 1):

- In der ersten Programmierungsphase 1995-1999 waren zwölf Programme allein für die regional-orientierten Ziele vorgesehen: Förderung von Regionen mit Entwicklungsrückstand (Burgenland, Ziel 1), vier regionale Programme zur Förderung von Industriegebieten mit rückläufiger Entwicklung (Ziel 2), sieben Programme zur Förderung von ländlichen Gebieten (Ziel 5b). Darüber hinaus kamen vier nationale Programme im Bereich der Arbeitsmarktpolitik sowie der ländlichen Entwicklung zur Umsetzung. Neben grenzüberschreitenden Räumen im Rahmen der INTERREGFörderung (sechs Programme) kam es noch zur kleinräumigen Abgrenzung einer Reihe von Gemeinschaftsinitiativen wie RESIDER, RECHAR, RETEX, URBAN sowie von ländlichen Kleinregionen im Rahmen der LEADER-II-Förderung. Mehr als $60 \%$ des Bundesgebietes, in denen rund $41 \%$ der österreichischen Bevölkerung lebten, wurden als Fördergebiet der Strukturfonds abgegrenzt. Damit wurden praktisch alle ländlichen und industriellen Regionen Österreichs außerhalb der Zentralräume erfasst.

- Die Agenda 2000 führte in Österreich zur Mittelkonzentration in der Periode 20002006 sowie zu einer Reduktion der für Österreich relevanten EU-Ziele auf Ziel 1 (Förderung von Regionen mit Entwicklungsrückstand - in Österreich das Burgenland) und Ziel 2 (Regionen mit wirtschaftlichen und sozialen Strukturproblemen). Weiters wurde die Zahl der nationalen Programme im Bereich der Arbeitsmarktpolitik sowie der ländlichen Entwicklung auf zwei reduziert. Ergänzend kamen vier Gemeinschaftsinitiativen sowie Pilotmaßnahmen unter der Programmlinie „Innovative Maßnahmen des EFRE“ in den Ländern zur Umsetzung. Die räumlich orientierten Gemeinschaftsinitiativen wie INTERREG, URBAN oder LEADER wurden aufgewertet. Die Periode 2000-2006 brachte eine Reduktion der Fördergebietskulisse um fast ein Drittel (gemessen an der Einwohnerzahl) auf 27,5\% der Einwohner.

- Die Reform im Lichte der Lissabon-Strategie in der Periode 2007-2013 führte für Österreich zur Konzentration der Förderinhalte. Es wurden ein Programm Konvergenz-Phasing-Out Burgenland sowie acht regionale Programme zur regionalen Wettbewerbsfähigkeit in den Ländern zur Umsetzung gebracht. Darüber hinaus gab es weiterhin zwei nationale Programme im Bereich der Arbeitsmarktpolitik (ESF) sowie der ländlichen Entwicklung (ELER). Mit der Verschiebung von der Ausgleichszielsetzung hin zu einer potenzialorientierten Entwicklungs- und Wachstumsperspektive im Ziel „Regionale Wettbewerbsfähigkeit und Beschäftigung“ wurde das ,innerregionale Mikrozoning“ aufgegeben, d.h. dass das gesamte Bundesgebiet förderfähig wurde. Umgekehrt wurden die INTERREG-Folgeprogramme (nunmehr Programme der Europäischen Territorialen Zusammenarbeit, ETZ) inhaltlich und finanziell deutlich aufgewertet. LEADER wurde vollständig in das Entwicklungsprogramm „Ländlicher Raum“ integriert und damit von der EU-Kohäsionspolitik abgekoppelt.

- In der Periode 2014-2020 ist eine klare Orientierung der Programme an den Europa2020-Zielen und den damit korrespondierenden Zielen des Österreichischen Nationalen Reformprogrammes notwendig. Aufgrund der Erfahrungen der steigenden 
administrativen Berichts- und Kontrollerfordernisse wurden in Österreich die bislang neun regionalen Entwicklungsprogramme des EFRE in einem nationalen Programm mit dezentralen Umsetzungsstrukturen zusammengefasst. Insgesamt reduziert sich damit die Zahl der Programme deutlich auf vier nationale Programme für die Ziele „Investitionen in Wachstum“ und „Beschäftigung“ (jeweils ein Programm EFRE sowie ESF) sowie „Entwicklung des ländlichen Raumes“ (jeweils ein Programm für ELER und Fischereifonds). Ergänzend kommen weiterhin die ETZ-Programme zur Umsetzung. Die Zahl der Kleinregionen im Rahmen von CLLD (vormals LEADER) wird aufgrund der reduzierten Finanzmittel wieder zurückgenommen. Die fakultativ vonseiten der EU angebotenen neuen, auf integrierte Raumentwicklung ausgerichteten Instrumente werden nur sehr eingeschränkt aufgegriffen. Bislang ist in Österreich unter den an der Umsetzung der Strukturfonds Beteiligten eine große Skepsis aufgrund der bereits bestehenden Komplexität und der in vielen Bereichen unklaren Vorgaben der EK zu beobachten.

Damit zeigt sich anhand der Entwicklung in Österreich wie die ,architektonischen Verschiebungen" der EU-Kohäsionspolitik mitvollzogen wurden. Es erfolgten ein Übergang von regionalen Problemgebieten hin zur Förderfähigkeit des gesamten Bundesgebiets sowie eine inhaltliche Anpassung der Programme an die Lissabonbzw. Europa-2020-Ziele. Darüber hinaus wurden die verbleibenden, gebiets- und raumtypen-orientierten Gemeinschaftsinitiativen URBAN und LEADER in die Mainstreamprogramme integriert und einem „Mainstreaming“ unterzogen. De facto bedeutete dies jedoch letztlich eine Schwächung dieser Instrumente. Die programmatische Konzentration mündet in vier nationalen Programmen, welche jeweils auf die zugehörigen Fonds ausgerichtet sind, sowie in die weiterhin bestehenden Programme der Europäischen Territorialen Kooperation.

Österreich verfügt als föderaler Staat über gute Voraussetzungen für eine integrierte territoriale Entwicklung. Die Größe der Länder erscheint als eine geeignete räumliche Abstimmungsebene für den Einsatz von entwicklungsfördernden Instrumenten. Österreich hat frühzeitig einen territorial orientierten Politikansatz verfolgt, der bereits in den auf den Erfahrungen der endogenen Erneuerung basierenden Entwicklungskonzepten ab Ende der 1980er Jahre erkennbar ist. Nicht zuletzt angestoßen durch die EU-Programmierungszyklen und die damit verbundenen programmatischen Arbeiten sowie im Zusammenhang mit einer insgesamt zunehmenden strategischen Ausrichtung der Interventionen begannen die Länder ab den 1990er Jahren Entwicklungsstrategien aufzulegen. Diese berücksichtigen die übergeordneten EU- und nationalen Politiken und spezifizieren regionale Stärken. Form, Gestaltung, Planungszeitraum und inhaltliche Ausrichtung orientieren sich dabei an den regionalen Gegebenheiten. Diese Strategien bilden die Grundlage für die EU-Programme.

Gerade der neue österreichische Ansatz von nationalen Programmen mit dezentralen Umsetzungskomponenten im EFRE stellt eine interessante Variante für die Sicherstellung von Territorialität dar, die internationale Beachtung finden sollte. Die territoriale Dimension orientiert sich in diesem System allerdings vorwiegend an Verwaltungsgrenzen (von Ländern) und nicht an Gebietstypen und deren Problemlagen und Potenzialen. Dies spiegelt sich auch deutlich in den Entwürfen der österreichi- 


\begin{tabular}{|c|c|c|}
\hline Periode & Periode 1: 1995-1999 & $\begin{array}{l}\text { Periode 2: 2000-2006 } \\
\text { (Reform Agenda 2000) }\end{array}$ \\
\hline $\begin{array}{l}\text { SF- } \\
\text { Fonds- } \\
\text { mittel }\end{array}$ & $\begin{array}{l}\text { 1,6 Mrd. Euro; } 25 \% \text { EFRE, } 37 \% \text { ESF, } \\
38 \% \text { EAGFL-A }\end{array}$ & $\begin{array}{l}\text { 1,98 Mrd. Euro; 55\% EFRE, 38\% ESF, } \\
6 \% \text { EAGFL-A }\end{array}$ \\
\hline Ziele & $\begin{array}{l}\text { Ziel 1: Förderung von Regionen mit } \\
\text { Entwicklungsrückstand: } 1 \text { Programm } \\
\text { Burgenland; } \\
\text { Ziel 2: Förderung von Industriegebie- } \\
\text { ten mit rückläufiger Entwicklung: } \\
4 \text { Programme Steiermark, Niederöster- } \\
\text { reich, Oberösterreich, Vorarlberg; } \\
\text { Ziel 3: Erleichterung der Eingliederung } \\
\text { am Arbeitsmarkt: } 1 \text { nationales Pro- } \\
\text { gramm; } \\
\text { Ziel 4: Erleichterung der Anpassung } \\
\text { der Arbeitskräfte: } 1 \text { nationales Pro- } \\
\text { gramm; } \\
\text { Ziel 5a: Erleichterung der Strukturan- } \\
\text { passung der ländlichen Gebiete: } \\
2 \text { nationale Programme; } \\
\text { Ziel 5b: Förderung von ländlichen Ge- } \\
\text { bieten: } 7 \text { Programme: Niederösterreich, } \\
\text { Oberösterreich, Steiermark, Salzburg, } \\
\text { Tirol, Kärnten, Vorarlberg }\end{array}$ & $\begin{array}{l}\text { Ziel 1: Förderung von Regionen mit } \\
\text { Entwicklungsrückstand: } 1 \text { Programm } \\
\text { Burgenland; } \\
\text { Ziel 2: Förderung von Regionen mit } \\
\text { wirtschaftlichen und sozialen Struktur- } \\
\text { problemen: } 8 \text { Ziel-2-Programme (inkl. } \\
\text { Phasing-Out Gebiete): alle Länder } \\
\text { außer Burgenland; } \\
\text { Ziel 3: Förderung der Anpassung und } \\
\text { Modernisierung der Bildungs-, Ausbil- } \\
\text { dungs- und Beschäftigungspolitiken: } \\
1 \text { nationales Programm; } \\
\text { EAGFL-G: } \\
\text { Entwicklung des ländlichen Raumes: } \\
1 \text { nationales Programm im Rahmen } \\
\text { des EAGFL-G (GAP) }\end{array}$ \\
\hline $\begin{array}{l}\text { Gemein- } \\
\text { schafts- } \\
\text { initiati- } \\
\text { ven }(G I)\end{array}$ & $\begin{array}{l}\text { INTERREG II (6 Programme), UR- } \\
\text { BAN (Wien, Graz), KMU, RETEX } \\
\text { (Niederösterreich, Steiermark, Vor- } \\
\text { arlberg), RESIDER (Oberösterreich, } \\
\text { Steiermark), RECHAR (Steiermark), } \\
\text { LEADER II (8 Programme), EM- } \\
\text { PLOYMENT, ADAPT (jeweils } \\
1 \text { Programm) }\end{array}$ & $\begin{array}{l}\text { INTERREG III (7 Programme), } \\
\text { URBAN (Graz, Wien), LEADER+ } \\
\text { (1 nationales Programm), EQUAL } \\
\text { (1 nationales Programm); Pilotaktion: } \\
\text { innovative Maßnahmen des EFRE } \\
\text { (in allen Ländern) }\end{array}$ \\
\hline $\begin{array}{l}\text { Charak- } \\
\text { teristik }\end{array}$ & Integrierte Programme & $\begin{array}{l}\text { Ziel-Programme, zum Teil von mehre- } \\
\text { ren Fonds gespeist, GI als Monofonds- } \\
\text { programme }\end{array}$ \\
\hline $\begin{array}{l}\text { Zielge- } \\
\text { biete }\end{array}$ & $\begin{array}{l}40,8 \% \text { Bevölkerungsanteil in Zielge- } \\
\text { bieten }\end{array}$ & $\begin{array}{l}27,5 \% \text { Bevölkerungsanteil in Zielge- } \\
\text { bieten }\end{array}$ \\
\hline
\end{tabular}

Quelle: Aktualisiert nach GrubER 2009

Tab. 1: Übersicht zu den vier Förderperioden in Österreich 


\begin{tabular}{|c|c|c|}
\hline Periode & $\begin{array}{l}\text { Periode 3: 2007-2013 } \\
\text { (Reform: Lissabon-Agenda) }\end{array}$ & Periode 4: 2014-2020 (Europa 2020) \\
\hline $\begin{array}{l}\text { SF- } \\
\text { Fonds- } \\
\text { mittel }\end{array}$ & 1,46 Mrd. Euro; 64\% EFRE, 36\% ESF & $\begin{array}{l}\text { Rund } 1 \text { Mrd. Euro EFRE, ESF, } \\
\text { 3,9 Mrd. Euro ELER, rund } 7 \text { Mio. } \\
\text { Euro EMFF }\end{array}$ \\
\hline Ziele & $\begin{array}{l}\text { Ziel „Konvergenz/Phasing Out“: je } \\
1 \text { EFRE bzw. ESF-Programm Burgen- } \\
\text { land; } \\
\text { Ziel „Regionale Wettbewerbsfähigkeit } \\
\text { und Beschäftigung: } 8 \text { EFRE-Program- } \\
\text { me: alle Länder außer Burgenland; } \\
1 \text { nationales ESF-Programm; } \\
\text { Ziel „Europäische Territoriale Zusam- } \\
\text { menarbeit (ETZ): } 7 \text { Programme für } \\
\text { grenzüberschreitende Zusammenarbeit } \\
\text { mit den Nachbarländern Tschechien, } \\
\text { Slowakei, Ungarn, Slowenien, Italien, } \\
\text { Alpenrhein-Bodensee, Bayern (D) } \\
\text { sowie Teilnahme an transnationalen } \\
\text { Programmen; } \\
\text { außerhalb der Strukturfonds: } 1 \text { natio- } \\
\text { nales Programm Entwicklung des } \\
\text { ländlichen Raumes (ELER) }\end{array}$ & $\begin{array}{l}\text { Ziel: Investitionen in Wachstum und } \\
\text { Beschäftigung - } 1 \text { nationales Pro- } \\
\text { gramm für EFRE sowie } 1 \text { nationales } \\
\text { Programm für ESF; } \\
\text { Entwicklung des ländlichen Raumes } \\
\text { - jeweils } 1 \text { nationales Programm für } \\
\text { ELER und EMFF (Fischereifonds); } \\
\text { Europäische Territoriale Zusam- } \\
\text { menarbeit (ETZ): } 7 \text { Programme für } \\
\text { grenzüberschreitende Zusammenarbeit } \\
\text { mit den Nachbarländern Tschechien, } \\
\text { Slowakei, Ungarn, Slowenien, Italien, } \\
\text { Alpenrhein-Bodensee, Bayern (D) } \\
\text { sowie Teilnahme an transnationalen } \\
\text { Programmen; } \\
\text { inhaltliche Ausrichtung auf } 11 \text { für alle } \\
\text { kohäsionspolitischen Programme vor- } \\
\text { gegebene thematische Ziele; }\end{array}$ \\
\hline $\begin{array}{l}\text { Gemein- } \\
\text { schafts- } \\
\text { initiati- } \\
\text { ven (GI) }\end{array}$ & keine & keine \\
\hline $\begin{array}{l}\text { Charak- } \\
\text { teristik }\end{array}$ & $\begin{array}{l}\text { Monofondsprogramme mit strategi- } \\
\text { schem Dach des nationalen strategi- } \\
\text { schen Rahmenplanes }\end{array}$ & $\begin{array}{l}\text { Monofondsprogramme mit strategi- } \\
\text { schem Dach der nationalen Partner- } \\
\text { schaftsvereinbarung; enge Bindung an } \\
\text { das nationale Reformprogramm }\end{array}$ \\
\hline $\begin{array}{l}\text { Zielge- } \\
\text { biete }\end{array}$ & keine Zielgebietsabgrenzungen & keine Zielgebietsabgrenzungen \\
\hline
\end{tabular}


schen Programme zu Wachstum und Beschäftigung für den Europäischen Fonds für Regionale Entwicklung bzw. den Europäischen Sozialfonds wider. Eine der zentralen Herausforderungen besteht damit in der Überwindung einer an administrativen Grenzen orientierten Entwicklung. Eine konsequente Weiterentwicklung dieses Modells bedarf einer stärkeren Beachtung von funktionalen Räumen.

\section{Schlussfolgerungen}

Die „Territorialität“ in der EU-Kohäsionspolitik folgt einer wechselhaften Geschichte. Die im Jahr 1989 mit der Reform der Kohäsionspolitik und den damit verbundenen Strukturfonds eingeleitete Ausrichtung auf die Bewältigung territorialgebietstypischer Problemlagen ist in den Jahren der Lissabon-Strategie schrittweise zugunsten von Politikprioritäten, die mehr auf horizontale Ziele wie die Stärkung der Wettbewerbsfähigkeit und Wachstum ausgerichtet sind, und durch die Bindung an eine weitgehend ,enträumlichte` europäische Dachstrategie verloren gegangen. Im Hinblick auf die territoriale Dimension hat sich die EU damit immer mehr auf das Ziel der Europäischen Territorialen Kooperation zurückgezogen.

Zeitlich etwas asynchron zu diesem Rückzug haben sich die zwischenstaatlichen Diskussionen der Territorialen Agenda verstärkt. Dies mündete nicht zuletzt in die Aufnahme der territorialen Kohäsion als drittem Kohäsionsziel in den EU-Vertrag. Im Hinblick auf die EU-Kohäsionspolitik brachte dies neue Handlungsnotwendigkeiten. Gestärkt wurde dies durch die im BARCA-Bericht forcierte place-based policy und die neuen räumlich-territorialen Orientierungen der Smart Specialisation im Rahmen der Innovationsstrategien.

Nach der Phase einer De-Territorialisierungs-Tendenz in der Lissabon-Ära hat die territoriale Dimension in den EU-kohäsionspolitischen Verordnungen wieder einen deutlich stärkeren Niederschlag gefunden. Explizit angesprochen wird die Zielsetzung, mit den Fonds den wichtigsten territorialen Herausforderungen zu begegnen, wobei die Rollen von Städten, funktionalen Gebietseinheiten und Regionen mit spezifischen Problemen angesprochen werden. Neue spezifische Instrumente wie die Integrierte Territoriale Entwicklung oder der Ansatz des „Community Lead Local Developments“ wurden im EU-Regulativ konzipiert. Allerdings sind diese Vorhaben - mit wenigen Ausnahmen (wie dem städtischen Bereich) - fakultativ für die Mitgliedsstaaten. Hier wird erst eine Auswertung der aktuellen Programmgeneration zeigen, in welchem Ausmaß diese tatsächlich von den Mitgliedsstaaten aufgegriffen werden.

Insgesamt bleibt derzeit alles wenig verbindlich. Die Konzepte dienen vor allem als Leitbilder und haben den Charakter von Leitlinien. Häufig ist auch noch unklar (trotz ETZ), welche Instrumente (außer bei großen Infrastruktur-Netzen) wirklich eingesetzt werden und welche Rolle dabei die supranationale Ebene spielt. Letztlich entzieht sich der Begriff der „territorialen Kohäsion“ jedoch einer einfachen und verbindlichen Definition. Er ist mehr als Intention und Ausdruck eines politischen Willens zu verstehen, denn als objektiv beschreibbare Situation (SCHNEIDEWIND 2009). Generell kehrt die ,Territorialität‘ damit in Ansätzen in die Kohäsionspolitik zurück. 
Widersprüche zwischen großräumiger Kohäsion auf europäischer Ebene, der Instrumentalisierung für die Europa-2020-Ziele und territorial, regional und lokal orientierten Entwicklungsansätzen sind jedoch keineswegs aufgelöst.

\section{Literaturverzeichnis}

B ARCA F. (2009), An Agenda for a Reformed Cohesion Policy. A place-based approach to meeting European Union challenges and expectations. Independent report prepared at the request of Danuta Hübner, Commissioner for Regional Policy by Fabrizio Barca. Brussels.

Cooke Ph., Uranga M.G., Etexebarria G. (1997), Regional innovation systems: Institutional and organizational dimensions. In: Research Policy, 26, 4-5, S. 475-491.

Cooke P., Boekholt P., Tödtling F. (2000), The Governance of Innovation in Europe - Regional Perspectives on Global Competitiveness. London, Pinter.

Bundeskanzleramt (Hrsg.) (2011), Nationales Reformprogramm Österreich. Wien.

Europäische Kommission (Hrsg.) (1991), Europa 2000 - Perspektiven der künftigen Raumordnung der Gemeinschaft. Brüssel.

Europäische Kommission (Hrsg.) (1995), Europa 2000+ - Europäische Zusammenarbeit bei der Raumentwicklung. Brüssel.

EuropÄIsche Kommission (Hrsg.) (2008), Grünbuch zum territorialen Zusammenhalt. Territoriale Vielfalt als Stärke. Brüssel.

Europäische Kommission (Hrsg.) (2010), Europe 2020 - A strategy for smart, sustainable and inclusive growth, COM (2010) 2020, Brussels, 03.03.2010.

Europäische Kommission (Hrsg.) (2007), Leipzig Charter on Sustainable European Cities. Brussels.

Europäische Union (Hrsg.) (2011a), Territoriale Ausgangslage und Perspektiven der Europäischen Union. Ein Hintergrunddokument für die Territoriale Agenda der Europäischen Union. Brüssel.

EuropäIsche Union (Hrsg.) (2011b), Territoriale Agenda der Europäischen Union 2020. Für ein integratives, intelligentes und nachhaltiges Europa der vielfältigen Regionen. Brüssel.

Europäisches Parlament, Europäischer Rat (Hsrg.) (2013), Verordnung (EU) Nr. 1303/2013 des Europäischen Parlaments und des Europäischen Rates vom 17. Dezember 2013 mit gemeinsamen Bestimmungen über den Europäischen Fonds für regionale Entwicklung, den Europäischen Sozialfonds, den Kohäsionsfonds, den Europäischen Landwirtschaftsfonds für die Entwicklung des ländlichen Raums und den Europäischen Meeres- und Fischereifonds sowie mit allgemeinen Bestimmungen über den Europäischen Fonds für regionale Entwicklung, den Europäischen Sozialfonds, den Kohäsionsfonds und den Europäischen Meeres- und Fischereifonds und zur Aufhebung der Verordnung (EG) Nr. 1083/2006 des Rates.

Europäisches Parlament, EuropäIscher Rat (Hsrg.) (2013), Verordnung (EU) Nr. 1301/2013 des Europäischen Parlaments und des Europäischen Rates vom 17. Dezember 2013 über den Europäischen Fonds für regionale Entwicklung und mit besonderen Bestimmungen hinsichtlich des Ziels „Investitionen in Wachstum und Beschäftigung“ und zur Aufhebung der Verordnung (EG) Nr. 1080/2006.

European Commission (EC) (Hrsg.) (2012), Guide to Research and Innovation Strategies for Smart Specialisation. EU-Regional Policy. Luxembourg, Publications Office of the European Union. 
Gruber M. (2009), Strukturfondsförderung in Österreich - Eine Zwischen-Bilanz - Zusammenschau. In: ÖsterReICHISCHE RAUMORDNUNGSKONFERENZ (ÖROK) (Hrsg.), EU-Kohäsionspolitik in Österreich 1995-2007 - Eine Bilanz (= ÖROK-Schriftenreihe, 180), S. 10-37. Wien.

Gruber M., Zumbusch K. (2008), Partnerschaftliche Umsetzung der EU-Regionalpolitik in Österreich: Die Programmperioden 2000-2006 und 2007-2013. In: ÖstERREICHISCHE RAUMORDNUNGSKONFERENZ (ÖROK) (Hrsg.), 12. Raumordnungsbericht Österreichs. Wien.

Gruber M., Pohn-Weidinger S. (2012), Themen der Raumentwicklung und Regionalpolitik im Lichte der EU-Politiken. In: ÖsterReichische RaumordnungsKonfERENZ (ÖROK) (Hrsg.), 13. Raumordnungsbericht Österreichs. Wien.

Huber W. (2007), Top-down und/oder bottom-up? Österreichische Regionalpolitik zwischen EU-Förderprogrammen, Föderalismus und Eigen-Sinnigkeit der Subsysteme. CIPRAWorkshop „Planen, Steuern und Netzwerken in der Region“, Admont 25./26. Januar 2007. Konferenzpapier.

Hummelbrunner R., Gruber M., Pohn-Weidinger S. (2013), Ergebnisorientierung in der EUProgrammperiode 2014-2020, Anforderungen und Herausforderungen für Österreichs EFRE-Programme. Graz, im Auftrag der Österreichischen Raumordnungskonferenz (nicht publiziert).

ÖSTERREICHISCHE RAUMORDNUNGSKONFERENZ (ÖROK) (Hrsg.) (2012), STRAT.AT Bericht 2012. Zweiter Strategischer Bericht Österreich zur Umsetzung der EU-Kohäsionspolitik 2007-2013 (= ÖROK-Schriftenreihe, 188). Wien.

ÖsterReICHISCHE RAuMORDNUNGSKONFERENZ (ÖROK) (Hrsg.) (2014), EFRE-Programm Investitionen in Wachstum und Beschäftigung Österreich 2014-2020, Einreichfassung vom April 2014. Wien.

ÖSTERREICHISCHE RAUMORDNUNGSKONFERENZ (ÖROK) (Hrsg.) (2014), Investitionen in Wachstum und Beschäftigung Österreich 2014-2020 - Operationelles Programm für den Einsatz der EFRE-Mittel in Österreich, Einreichfassung. Wien.

Österreichische Raumordnungskonferenz (ÖROK) (Hrsg.), Informationen zur Partnerschaftsvereinbarung 2014-2020 zwischen der EK und Österreich. - http://www.oerok.gv.at/euregionalpolitik/europaeische-struktur-und-investitionsfonds-in-oesterreich-2014-2020/ partnerschaftsvereinbarung-stratat-2020/english-summary.html (zuletzt abgerufen 04.10.2014).

SCHNEIDEwind P. (2009), Die Ankunft der Räumlichkeit in der EU-Politik. In: Raum, 76, S. 46-49.

Zumbusch K., Hummelbrunner R., Pech S. (2009), Dreizehn Jahre EFRE-Förderungen in Österreich - Qualitative Effekte und Auswirkungen. In: ÖSTERREICHISCHE RAUMORDNUNGSKONFERENZ (ÖROK) (Hrsg.), EU-Kohäsionspolitik in Österreich 1995-2007 - Eine Bilanz (= ÖROK-Schriftenreihe, 180), S. 197-225. Wien. 